Mélanges

de l'École française de Rome

Moyen Âge
Mélanges de l'École

française de Rome - Moyen

Âge

128-2 (2016)

Dossier : « Ben più del denaro ». Il diritto della moneta, la sua sovranità, le sue funzioni Varia - Atelier doctoral - Regards croisés

Adele Di Lorenzo, Jean-Marie Martin et Annick Peters-Custot

\section{Le monastère de S. Elia di Carbone, ses archives et l'histoire de la Basilicate médiévale}

\begin{abstract}
Avertissement
Le contenu de ce site relève de la législation française sur la propriété intellectuelle et est la propriété exclusive de l'éditeur.

Les œuvres figurant sur ce site peuvent être consultées et reproduites sur un support papier ou numérique sous réserve qu'elles soient strictement réservées à un usage soit personnel, soit scientifique ou pédagogique excluant toute exploitation commerciale. La reproduction devra obligatoirement mentionner l'éditeur, le nom de la revue, l'auteur et la référence du document.

Toute autre reproduction est interdite sauf accord préalable de l'éditeur, en dehors des cas prévus par la législation en vigueur en France.
\end{abstract}

\section{revues.org}

Revues.org est un portail de revues en sciences humaines et sociales développé par le Cléo, Centre pour l'édition électronique ouverte (CNRS, EHESS, UP, UAPV).

\footnotetext{
Référence électronique

Éditeur : École française de Rome

http://mefrm.revues.org

http://www.revues.org

Document accessible en ligne sur :

http://mefrm.revues.org/3318

Document généré automatiquement le 07 septembre 2016.

(c) École française de Rome
}

Adele Di Lorenzo, Jean-Marie Martin et Annick Peters-Custot, « Le monastère de S. Elia di Carbone, ses archives et I'histoire de la Basilicate médiévale », Mélanges de l'École française de Rome - Moyen Âge [En ligne], 128-2 | 2016, mis en ligne le 07 septembre 2016, consulté le 07 septembre 2016. URL : http://mefrm.revues.org/3318 
Adele Di Lorenzo, Jean-Marie Martin et Annick Peters-Custot

\section{Le monastère de S. Elia di Carbone, ses archives et l'histoire de la Basilicate médiévale}

\section{Présentation générale}

Si l'Italie des communes, la République de Venise ou Rome ont longtemps concentré l'attention des historiens médiévistes spécialistes de la péninsule italienne, l'Italie méridionale et la Sicile médiévales sont désormais, et depuis quelques décennies, au cœur de renouvellements historiographiques profonds dans lesquels l'École française de Rome a joué et joue toujours un rôle indéniable, en soutenant et publiant les travaux de chercheurs qui se sont illustrés dans l'exploration de ce riche passé, depuis Henri Bresc ${ }^{1}$ et Jean-Marie Martin', jusqu'aux études issues de la génération suivante : Gian Luca Borghese ${ }^{3}$, Sulamith Brodbeck ${ }^{4}$, Philip Ditchfield ${ }^{5}$, Benoît Grévin ${ }^{6}$, Annliese Nef ${ }^{7}$, Annick Peters-Custot ${ }^{8}$ et bien d'autres encore ${ }^{9}$. Ces études ne doivent pas faire oublier le rôle essentiel, pour la recherche historique, des entreprises de publication des documents de l'Italie méridionale - dans lesquelles, là encore, l'École française de Rome a pris sa part ${ }^{10}$ - et, en particulier, celle des actes des monastères grecs de l'Italie du Sud byzantine, normande et souabe.

Le monachisme italo-grec constitue en effet un point d'ancrage historique fondamental dans l'étude des contacts entre Orient et Occident dans la Méditerranée médiévale, comme sur les rapports entre les formes monastiques et la société qui les produit, les réclame et les accueille, sur l'économie monastique, les effets de la conquête normande dans l'Italie byzantine, les phénomènes d'acculturation ou les usages politiques des enjeux de la diversité religieuse et culturelle. Notons que ces approches se jouent en large partie pour la période caractérisée, dans le reste de l'Occident, par la réforme pontificale et ses profondes conséquences socio-culturelles ${ }^{11}$, dont le monachisme byzantin tout comme l'Eglise italo-grecque restèrent longtemps protégés, grâce à la tutelle de leurs souverains ${ }^{12}$.

Ce monachisme grec d'Italie, qui s'était développé à Rome durant le haut Moyen Âge ${ }^{13}$, s'est largement répandu au-delà des frontières de l'Italie hellénisée et de l'Italie byzantine à la fin du $\mathrm{X}^{\mathrm{e}}$ siècle et, précisément, à partir des années 960-980. Il conviendrait du reste d'accorder une attention soutenue au mouvement migratoire assez massif de populations hellénophones — et notamment de moines - qui, partant de la Sicile et de la Calabre méridionale, ont essaimé vers le nord de la péninsule et se sont installées autour des années 960-980 et de manière concomitante à Rome, Naples, Salerne, Tarente, d'après les sources notariées et hagiographiques ${ }^{14}$. La domination islamique sur la Sicile est souvent invoquée comme cause unique de ces migrations précisément datées, dans la mesure où l'origine sicilienne et calabraise de cette population de langue grecque et de rite oriental est plus que probable ${ }^{15}$. Quoi qu'il en soit, le monachisme byzantin put ainsi s'implanter dans le Latium ${ }^{16}$, à Salerne ${ }^{17}$ et dans le Cilento ${ }^{18}$, voire dans l'espace germanique ${ }^{19}$. En Calabre et dans le Salento, le monachisme byzantin connut son apogée à l'époque dite «normande » et parallèlement reprit pied dans la Sicile des Hauteville ${ }^{20}$. Lieu de refuge majeur, mais non unique ${ }^{21}$, de l'héritage byzantin en Italie du Sud, le monachisme italo-grec résista ainsi à la séparation politique, à la fin du XI ${ }^{\mathrm{e}}$ siècle, d'avec l'Empire byzantin, et perdura jusqu'au Concile de Trente comme objet d'une attention soutenue de la part des souverains comme de la papautét ${ }^{22}$, au point de bénéficier de la rédaction d'une règle composée au milieu du $X V^{e}$ siècle, de la main de Bessarion, sur la base des écrits ascétiques de saint Basile le $\mathrm{Grand}^{23}$, dans la perspective de la création d'un «Ordre de saint Basile ${ }^{24}$. Ces moines et ces établissements, dont l'existence tend à saper les fondements d'une dichotomie rigide entre Orient et Occident, trop souvent avancée pour le Moyen Âge, devinrent les rédacteurs et conservateurs d'archives dont on 
saisit désormais l'ampleur quantitative et qualitative. Ainsi, c'est en grande partie grâce à la présence monastique grecque dans l'Italie médiévale que la documentation notariée italogrecque constitue, après les archives de l'Athos, le deuxième plus grand fonds médiéval en langue grecque - et même le premier pour le Moyen Âge central ${ }^{25}$. Après André Guillou, qui publia une partie non négligeable de ce $\operatorname{corpus}^{26}$, Cristina Rognoni poursuit l'entreprise éditoriale en publiant des actes privés grecs du monastère archimandrital du Saint-Sauveur de Messine qui sont relatifs à la Calabre ${ }^{27}$. Les fonds inédits sont désormais rares, mais il reste que certaines archives ne sont accessibles que dans des éditions anciennes, qui mériteraient d'être reprises. Il en est ainsi des actes grecs de l'abbaye de la Sainte-Trinité de Cava de' Tirreni, près de Salerne, publiés en 1865 par Francesco Trinchera ${ }^{28}$, et qui ont fait l'objet d'une attention récente tout à fait bienvenue ${ }^{29}$. Un autre fonds a été particulièrement maltraité par une édition incomplète et qui a beaucoup vieilli - elle était déjà dépassée du point de vue des critères scientifiques d'édition au moment même de sa parution : celui des actes grecs du monastère byzantin de S. Elia di Carbone, publiés par une Anglaise peu connue, Gertrude Robinson $^{30}$. Cette situation, dommageable à la connaissance d'un monastère et d'une population grecs immergés dans un environnement latin (la Basilicate méridionale et la région de Tarente) peut être réparée par une entreprise ambitieuse de réédition de ces documents grecs, produits aux $\mathrm{XI}^{\mathrm{e}}$ et $\mathrm{XII}$ e siècles, associée à la publication des actes latins du fonds, d'un nombre presque équivalent. L'ensemble offre en effet un inestimable aperçu des relations entre populations grecques et latines dans la Basilicate médiévale, et de la place sociale, politique et religieuse d'un monastère byzantin devenu en 1168, par la grâce royale, archimandritat, et en outre puissant centre économique, culturel et spirituel d'une enclave grecque de la Basilicate méridionale.

\section{Brève histoire du monastère}

Le monastère de Carbone, dédié d'abord à saint Anastase, puis aux saints Anastase et Elie, et enfin à saint Elie seul, est un établissement fondé à l'époque de la domination byzantine sur la Basilicate méridionale. D'après la fragile chronologie de ses débuts qu'il est permis de reconstituer notamment à partir du testament d'un des premiers higoumènes de l'établissement, lequel atteste les aléas initiaux largement liés aux incursions arabes ${ }^{31}$, le monastère de Carbone a été fondé à la fin du $\mathrm{X}^{\mathrm{e}}$ siècle par un disciple de Sabas de Collesano. Il relève donc de ces fondations grecques qui accompagnèrent le mouvement migratoire italo-grec déjà mentionné plus haut, et qui s'épanouirent entre autres dans deux régions, le Mercourion (c'est-à-dire la vallée du Lao) et le Latinianon (celle de Carbone), deux zones depuis peu reconquises sur les Lombards par les forces impériales ${ }^{32}$. Le peuplement grec, dans ces zones montagneuses, boisées et initialement sous-peuplées, conduisit à la constitution d'une sorte d'enclave ${ }^{33}$ où on produisait des documents notariés en grec, on suivait le droit et les usages socio-économiques byzantins ${ }^{34}$, et où ont fleuri des établissements monastiques orientaux, le plus souvent de petite taille; c'est pourquoi les hagiographies attribuèrent avec quelque complaisance le nom d'éparchie à ces zones, par référence aux mythiques éparchies monastiques d'Égypte et de Palestine ${ }^{35}$. Cette poche grecque en Basilicate méridionale se trouva consolidée a posteriori mais peu après par des initiatives administratives du pouvoir byzantin, et en particulier la fondation d'évêchés rattachés à Constantinople ${ }^{36}$. La poche italogrecque de Basilicate méridionale fut appelée à durer, malgré l'établissement, au cours du $\mathrm{XI}^{\mathrm{e}}$ siècle, de seigneurs normands qui firent bénéficier S. Elia di Carbone de leurs largesses, ce qui valut au monastère de devenir le plus important et le plus riche établissement grec de la Basilicate au XII ${ }^{e}$ siècle. Consécration de cette position éminente, le roi Guillaume II renforça la position de S. Elia di Carbone en 1168, en concédant à l'higoumène le titre et la fonction d'archimandrite de tous les monastères de Lucanie qui suivaient la « règle de saint Basile $»^{37}$, et qui étaient situés dans une vaste zone regroupant la plupart des monastères grecs locaux $^{38}$. L'archimandritat de Carbone diffère donc de celui du Saint-Sauveur de Messine non seulement par la personne de son fondateur et sa date de fondation, mais aussi par ses modalités — beaucoup moins claires — de définition : si l'higoumène du Saint-Sauveur a 
une autorité archimandritale sur des monastères dûment inventoriés, situés en Sicile comme en Calabre ${ }^{39}$, l'archimandritat de Carbone relève d'une domination territorialisée, dont on précise les confins.

La suite de l'histoire est classique dans le cadre du royaume de Sicile : S. Elia di Carbone, encore rayonnant aux XII ${ }^{\mathrm{e}}$ et XIII ${ }^{\mathrm{e}}$ siècles, amorce une lente phase de déclin, dont il convient de nuancer le rythme et les effets, jusqu' au début du XIV ${ }^{\mathrm{e}}$ siècle : à cette époque, l'établissement est encore suffisamment attractif pour susciter la vocation, en 1315, d'un prêtre latin du nom d'Acursus, devenu moine sous le nom de Grégoire, mais à qui on confie un prieuré proche, car il est incapable de suivre la liturgie en grec, d'après le memoratorium oblationis qui consigne son entrée au monastère ${ }^{40}$ - ce qui reflète le respect continu, encore à l'époque, de la liturgie byzantine à S. Elia di Carbone mais aussi, peut-être, le niveau économique encore plus dégradé des établissements religieux « latins » dans la région. À la même époque se clôt le long conflit qui oppose le monastère de Carbone à l'évêque d'Anglona (successeur latin de l'évêché grec de Tursi, fondé par Nicéphore Phocas) au sujet du rattachement juridictionnel du premier. En effet, le roi Guillaume II avait transféré Carbone sous la juridiction du nouvel évêché sicilien de Monreale, soustrayant ainsi l'établissement à l'ordinaire, à savoir l'évêque d'Anglona. S'en était suivi un conflit de plusieurs siècles, réglé dans les années $1320^{41}$.

Le déclin général que connut le monachisme traditionnel en Occident, à partir du XIV ${ }^{\mathrm{e}}$ siècle, était ici aggravé par les conflits guerriers incessants qui secouèrent l'Italie méridionale à partir des Vêpres siciliennes et, dans le cas spécifique du monachisme que la papauté désigne de plus en plus comme soumis à «l'Ordre de saint Basile » ${ }^{42}$, par le déclin de la langue grecque, aux conséquences liturgiques néfastes. Un inventaire tardif de la bibliothèque de $\mathrm{S}$. Elia de Carbone mentionne un « vocabolario greco » et des grammaires grecques, sans doute à l'usage des moines italo-grecs peu formés, mais aussi des recrues non grecques du monastère ${ }^{43}$. Lors de la visite des monastères basiliens, ordonnée en 1457 par le cardinal Bessarion et confiée à Athanase Chalkéopoulos, le monastère compte un archimandrite, Placide, à ce poste depuis $1432^{44}$, et six frères, Romain, Daniel, Pierre, Athanase, Laurent et Nicodème ${ }^{45}$. Si le comportement de l'archimandrite paraît nuire à la conservation des biens de l'établissement - en particulier parce qu'il dépouille régulièrement le monastère de ses biens au profit de ses enfants - les frères affirment que leur supérieur récite chaque jour les offices, et l'inventaire des livres de la bibliothèque comprend un nombre confortable de volumes surtout en comparaison des pauvres listes bien souvent dressées à cette occasion pour les autres établissements visités ${ }^{46}$. S. Elia di Carbone est concédé en commende (1474); nous verrons le rôle joué par les abbés commendataires dans la conservation des documents du monastère.

\section{Le fonds de Carbone : caractères originaux}

On sait que le monastère de S. Elia de Carbone n'existe plus, mais ses archives sont conservées, pour la plus grande partie, à Rome à l'Archivio Doria Pamphili ${ }^{47}$. Les parchemins sont entrés dans cet Archivio romain vers 1640 grâce à l'abbé commendataire Giovanni Battista Pamphili, futur pape Innocent $X^{48}$.

Le fonds de l'abbaye, qui constitue un patrimoine unique pour l'étude de l'histoire et de la culture du territoire compris entre l'Agri et le Sinni, commence au $\mathrm{XI}^{\mathrm{e}}$ siècle et va jusqu'à l'époque moderne. Il éclaire l'activité économique et sociale d'une zone marquée, on l'a dit, par une forte présence grecque issue d'une migration massive, attestée dès le $\mathrm{IX}^{\mathrm{e}}$ siècle, venue de la Calabre méridionale et de la Sicile ${ }^{49}$.

Pour l'époque byzantine et normande, il a l'intérêt de contenir des actes grecs, latins et bilingues (deux préceptes bilingues, en grec et en latin, de Guillaume II et de Constance).

Pour l'époque prénormande on ne trouve que huit actes grecs ; en revanche, de 1074 à 1194 on trouve 60 actes (originaux ou copies), dont 42 originaux grecs, 4 traductions latines d'originaux perdus ${ }^{50} ; 13$ actes latins, un privilège bilingue de Guillaume II. Trois documents sont conservés hors de l'Archivio Doria Pamphili : une donation d'Arnaud de Craco (1105), le privilège de Jean évêque de Marsico (1134), la confirmation des possessions faite par Néel frère d'Alexandre de Chiaromonte (1102), conservée en copie moderne sur une feuille ajoutée 
à l'appendice documentaire du Chronicon Carbonense de Pietro Menniti, dont on reparlera ${ }^{51}$. Avec l'apport de ces trois derniers documents, on arrive à un total de 47 actes grecs et 15 latins. De la conquête normande à l'arrivée des Souabes, les archives du monastère de Carbone conservent 8 parchemins latins, établis sur le territoire compris entre l'embouchure du Sinni et Tarente ; quatre autres ont des provenances différentes : Salerne (1159), S. Sofia près de Lavello (1168), Messine (1168), Bari (1171), sans compter l'acte déjà cité de l'évêque de Marsico $^{52}$.

En fait, les documents latins du bas Moyen Âge et du $\mathrm{XVI}^{\mathrm{e}}$ siècle sont nombreux. Gastone Breccia a donné des régestes des documents établis entre 1276 et $1473^{53}$; il en compte 30 ( 5 de la fin du XIII ${ }^{\mathrm{e}}$ siècle, 7 de la première moitié et 4 de la seconde moitié du XIV ${ }^{\mathrm{e}}$ siècle, $14 \mathrm{du} \mathrm{XV}$ e siècle). Le fonds se termine avec quelques actes du $\mathrm{XVI}^{\mathrm{e}}$ siècle.

$\mathrm{Si}$ on se réfère aux seuls parchemins latins (et deux bilingues) conservés à l'Archivio Doria Pamphili, on trouve les chiffres (approximatifs) suivants : 2 actes du XI ${ }^{\mathrm{e}}$ siècle ; $20 \mathrm{du} \mathrm{XII}{ }^{\mathrm{e}}$ (dont 5 insérés dans des actes postérieurs) ; 9 du XIII (dont deux insérés) ; 11 du XIV ${ }^{\mathrm{e}}, 6 \mathrm{du}$ $\mathrm{XV}^{\mathrm{e}}$ et $6 \mathrm{du} \mathrm{XVI}^{\mathrm{e}}$. Deux parchemins ( ${ }^{\text {os }} 10$ et 25$)$ ne concernent pas Carbone.

Du point de vue économique, les documents du fonds de Carbone témoignent de la vitalité des petits monastères ruraux de la Basilicate orientale, comme en attestent les testaments et les donations pro anima conservés dans les actes grecs ; ils sont également révélateurs du caractère agricole des activités économiques des monastères de ce territoire, de la forte présence des familles des fondateurs et aussi, d'après l'analyse des souscriptions des fonctionnaires dans les donations, d'un lien étroit avec les autorités administratives impériales ${ }^{54}$. Le monastère de Carbone lui-même appartenait au même type de fondation rurale, dont la vie fut fortement affectée par de nombreuses incursions, come par l'avidité des voisins. Sous l'abbatiat de Blaise, frère de l'higoumène Luc, qui prit la direction du monastère en 1058/59, les Normands avaient déjà soumis presque toute la Basilicate. Après la conquête, dans le cadre de la politique de restauration des fondations et d'augmentation des ressources des grands centres monastiques suivie par les souverains, d'après un acte édité par Holtzmann, l'abbé grec du monastère de Carbone cherche la protection des nouveaux seigneurs en la personne d'un représentant des familles puissantes de la région, Hugues de Chiaromonte ${ }^{55}$. Du point de vue linguistique, les documents présentent une situation plutôt claire et simple au sein d'une Basilicate hétérogène : aux XI ${ }^{\mathrm{e}}$ et XII ${ }^{\mathrm{e}}$ siècles, l'usage de la langue grecque persiste jusqu'à la fin de l'époque normande sur les cours moyens et inférieurs de l'Agri et du Sinni ; le monastère de $\mathrm{S}$. Elia est un puissant bastion de cet hellénisme, à cause sans doute de la position centrale dont il jouit : la vallée du Sinni le met en communication avec la côte ionienne et Tarente. L'étude des archives de Carbone montre que, de 1043 à 1183, l'usage de la langue greque persiste dans trois aires géographiques. La première est celle que traverse le Sinni : Carbone, Teana, Noepoli, Senise ; la seconde est celle de l'Agri, avec les centres de Viggiano, Sant'Arcangelo, Policoro ; la troisième se trouve au sud du Sinni, en direction de la Calabre, avec Oriolo et Laino et, plus au sud, Mormanno et Cerchiara di Calabria. Tarente apparaît dans le dossier avec un ensemble de 15 documents, majoritairement grecs, qui couvrent plus d'un siècle (1049-1198) ${ }^{56}$. Comme on ne connaît pas de donations faites au monastère par des représentants de la population locale après $1071^{57}$, on peut affirmer que c'est le lien entre Carbone et Tarente qui garantissait la survivance de la langue grecque ; en effet, une classe moyenne tarentine de culture et de formation grecques a survécu pendant tout le XII ${ }^{\mathrm{e}}$ siècle $^{58}$. Bastion de l'hellénisme dans le sud-est de la Basilicate au moins jusqu'à la fin du règne de Roger II, les archives de Carbone présentent un caractère bilingue qui constitue l'un des aspects les plus originaux de ce fonds monastique. Par rapport aux grecs, les documents latins sont plutôt rares (13) et ont été faits surtout le long de la vallée du Sinni, à Chiaromonte, Senise, Colobraro, et sur l'Agri à Marsico. Le plus ancien est daté de 1074 (donation de Hugues de Chiaromonte et de son épouse Giamarga) et montre l'utilisation de notaires ou de chapelains proches de la famille normande (en réalité française) récemment implantée ; rapidement on passera à la pratique linguistique et notariale du territoire de Carbone ; en 
revanche la diplomatique latine prend forme plus tard, si on se réfère aux actes de Senise et de Colobraro ${ }^{59}$.

Bien qu'on observe une hétérogénéité objective dans la diplomatique latine, il faut considérer ce fonds précieux comme un ensemble qui ne présente pas d'élément marginal, mais une pluralité dynamique d'expériences linguistiques inspirées de territoires de référence : Tarente pour les notaires grecs, les zones plus restreintes des vallées du Sinni et du Serropotamo pour les latins - Richard, chapelain au service d'Hugues de Chiaromonte (1074), le prêtre Jean Craco (1105), Roger notaire de Senise (1170), Porphyre notaire de Senise $(1172,1189)^{60}$. Il n'y a pas lieu de parler ici de la diplomatique latine du bas Moyen Âge, normalisée par la monarchie au XIII ${ }^{\mathrm{e}}$ siècle (généralisation du memoratorium).

Les éditions existantes - toutes partielles - sont en outre encore incomplètes, surtout si on tient compte de la multiplication des centres d'intérêt concernant ce type d'étude : les aspects paléographiques et codicologiques, mais aussi les données proprement juridiques concernant notamment le formulaire des donations. Il s'agit en effet d'éditions faites à une époque où l'analyse diplomatique ne cherchait pas à approfondir les modes de composition du texte. Aujourd'hui nous savons que l'étude de la forme des actes fournit un indice du niveau culturel de ceux qui les établissent, et non seulement de l'idéologie des auteurs des documents.

\section{Éditions antérieures et travaux : l'histoire du fonds}

Les matériaux pour l'histoire médiévale du monastère de Carbone sont à la fois riches et complexes. Ce grand monastère, qui n'a été supprimé qu'en $1809^{61}$, a suscité l'intérêt des savants pendant l'époque moderne, du milieu du XVI ${ }^{\mathrm{e}}$ au début du XVIII siècle, alors qu'il était en pleine décadence. Les abbés commendataires et les abbés généraux de l'ordre de saint Basile ont alors su conserver, traduire ou copier de nombreux documents d'archives, mais nous les ont transmis dans un certain désordre. L'abbaye avait jusqu'alors conservé (en dépit des incendies qui l'ont frappée en 1177 et en $1432^{62}$ ) un bon nombre de documents, et aussi de livres dont certains sont encore conservés à la Bibliothèque Vaticane et à Grottaferrata ${ }^{63}$. Quant aux documents, ils sont aujourd'hui dispersés entre l'Archivio Doria Pamphili, le fonds «Basiliani » de l'Archivio Segreto Vaticano, et des éditions anciennes de documents aujourd'hui disparus. C'est aux XVII' et XVIII ${ }^{e}$ siècles que s'est produite, en plusieurs temps, cette dispersion, qui a du moins permis de sauvegarder une bonne partie des archives.

C'est d'abord à trois abbés commendataires, les cardinaux Giulio Antonio Santoro, Paolo Emilio Santoro (neveu du précédent) et Giovanni Battista Pamphili ${ }^{64}$, dont les abbatiats couvrent la période 1570-1644, que nous devons la conservation, sous plusieurs formes, du fonds de Carbone.

La plus grande partie des archives conservées (notamment tous les originaux) se trouve aujurd'hui à l'Archivio Doria Pamphili, à Rome. Elles y ont été transférées, semble-t-il, par le cardinal Giovanni Battista Pamphili (futur pape Innocent X), nommé en 1630 abbé commendataire de l'abbaye de Carbone et cardinal protecteur de l'ordre basilien. Mais ces archives devaient déjà se trouver à Rome depuis la fin du XVI siècle : Holtzmann fait remarquer ${ }^{65}$ qu'une partie au moins du fonds y était en 1581, date à laquelle le crucigère Nicolaus Stridonius est cité comme traducteur d'un acte (Robinson XXXI) ; on peut ajouter que le cardinal Paolo Emilio Santoro, dont le livre (sur lequel on va revenir) a été publié en 1601 , avait dû voir à Rome les documents qu'il y publie. On ne peut donc suivre avec précision le chemin des archives de Carbone jusqu'à l'Archivio Doria Pamphili. Elles y étaient en tout cas au début du XVIII ${ }^{\mathrm{e}}$ siècle, puisque Pietro Menniti situe un certain nombre d'actes à la $B . P$. (Bibliotheca Pamphili). Là se trouve aujourd'hui, en tout cas, la totalité des documents grecs conservés, publiés par Gertrude Robinson (qui indique pour chacun, en chiffres arabes, leur cote à l'Archivio Doria Pamphili).

21 Mais l'histoire de la publication des archives de l'abbaye commence avant leur transfert dans cet Archivio. La première date à retenir est en effet celle de 1601, date de l'impression de l'Historia monasterii Carbonensis de Paolo Emilio Santoro, futur archevêque de Cosenza (1617-1623), puis d'Urbino (1623-1633). Il était neveu du cardinal Giulio Antonio Santoro 
que Pie V avait nommé en 1570 abbé commendataire de Carbone, et qui mourut en 1602 ; à la mort de son oncle, Paolo Emilio devait lui succéder comme abbé commendataire de Carbone et devenir aussi cardinal protecteur de l'ordre basilien ${ }^{66}$; il eut, à son tour, pour successeur dans cette double charge le cardinal Giovanni Battista Pamphili, dont on a déjà évoqué le rôle. Mais revenons aux cardinaux Santoro : le premier a entrepris (et le second a continué, apparemment de 1601 à 1612) de faire traduire en latin un certain nombre d'actes grecs par Federico Metio (futur évêque de Termoli) ${ }^{67}$; d'autres traductions, on l'a vu, avaient déjà été faites ${ }^{68}$.

L'Historia monasterii Carbonensis de Paolo Emilio Santoro, qui reprend certaines de ces traductions, est un livre de petit format, de 228 pages $^{69}$. Le récit historique de Santoro est assez décevant. Il s'ouvre sur une liste des archimandrites (p. 3-5), qui commence par le fondateur supposé, «Sanctus Magnus Lucas cognomento Carboni Magni Sabae discipulus et socius » et est suivie de la liste des commendataires (p. 5), jusqu'à l'oncle de l'auteur (mort en 1602 et donc encore en poste lors de la parution du livre) ; viennent ensuite (p. 5-10) les nomina benefactorum. Le récit proprement dit commence à la p. 13 ; il expose dès l'abord que le Carbonense monaterium, ordinis S. Basilii a été édifié « in Lucania opimo solo provincia, et uberi grege, armentisque »; il est dédié à la Mère de Dieu, à saint Anastase le Perse et à Élie. L'auteur reconnaît que l'origine de l'abbaye est incertaine, mais est attribuée au Calabrais Luc, qui a été moine en Sicile à S. Filippo d'Agira, puis ermite dans une grotte en Calabre ; il aurait ensuite passé sept ans à Noa (Noepoli) dans une église St-Pierre, se serait déplacé à Grumento où il aurait bâti une petite église St-Julien et se serait fait remarquer par ses aumônes qui l'apparentaient au patriarche d'Alexandrie saint Jean l'Aumônier. Ayant subi les attaques d'un baron Landolf, il aurait enfin fondé le monastère de Carbone, y attirant des moines inorganisés d'Armento. Il aurait encore résisté aux Sarrasins qui avaient saccagé une église de la Vierge près d'Armento. Enfin il serait mort le 13 octobre 993 en présence de Sabas et aurait été enterré à Armento (p. 14-24) ${ }^{70}$.

Son quatrième successeur, Blaise, parti pour Jérusalem, aurait rapporté d'Orient le corps de sainte Marie l'Égyptienne et la tête de saint Jean l'Aumônier, et aurait édifié une église StLuc. Le corps de sainte Marie l'Égyptienne aurait été emporté à Naples (au XIV siècle) par la reine Sancha, mais le monastère aurait gardé le chef de saint Jean l'Aumônier, pour lequel l'oncle de l'auteur a fait faire une châsse. Carbone aurait encore reçu des reliques de saint Guy (san Vito) et de saint Christophe (p. 24-28). L'abbatiat de Nil de Rossano, disciple du célèbre saint homonyme (au début du XII ${ }^{e}$ siècle) permet à l'auteur une digression sur le fondateur de Grottaferrata.

La suite du récit est une assez morne compilation d'histoire générale, dans laquelle sont insérés les faits marquants de l'histoire de l'abbaye. Dans la dernière partie, il insiste sur les luttes contre les Sanseverino de Bisignano (qualifiés de reguli) et sur les commendataires, dont l'action est en général jugée négative, à quelques exceptions près (récupération d'Asprella par Iacobus Ferrarius en 1459, p. 123). Mais l'histoire s'achève heureusement sur l'action de l'actuel commandataire, dont la vie est retracée (p. 193-201); il a notament fait traduire des documents grecs en latin par Federico Metio (p. 204).

Le principal intérêt du livre de Paolo Emilio Santoro est qu'il publie intégralement, dans le cours de son récit, 17 actes du fonds de Carbone, dont certains ont été ensuite perdus. Mais il n'édite que des documents latins, originaux ou traductions du grec faites par Metio. Donnons-en une liste rapide :

27 1. P. 52-53 : Roger II. 2. P. 56-58 : Alexandre de Chiaromonte. 3. P. 61-65 : Bohémond II. 4. P. 67-68 : Guillaume I ${ }^{\text {er }}$ 5. P. 84-85 : Tancrède (roi de Sicile). 6. P. 91-94 : Frédéric II. 7. P. 111-115 : Hugues de Chiaromonte. 8. P. 115-121 : memoratorium fait à Carbone le 7 février 1473. 9. P. 146-150 : Bohémond II. 10. P. 150-153 : Richard le Sénéchal. 11. P. 153-159: Alexandre et Richard de Chiaromonte. 12. P. 161-162 : jugement de la chambre de la Summaria. 13. P. 173-174 : bulle d'Innocent [V]. 14. P. 174-175 : bulle d'Innocent [VI]. 15. P. 175-178 : bulle de Boniface [IX]. 16. P. 179-185 : Ferdinand d'Aragon. 17. P. 207-211: jugement de Pie V sur un litige entre Carbone et l'évêque d'Anglona.

Une étude précise sera nécessaire pour distinguer d'une part les documents qui ne nous ont été transmis que par Santoro de ceux dont nous possédons encore les originaux, d'autre part 
les copies d'originaux latins et les copies de traductions grecques. Le fonds Doria Pamphili contient en effet de nombreux originaux latins inédits. Holtzmann a repéré et publié deux versions légèrement différentes d'un acte de 1074 transmis (sans doute en traduction latine) d'une part par un parchemin de la fin du XII ${ }^{\mathrm{e}}$ siècle, d'autre part par Santoro (repris par Ughelli) $^{71}$. De fait, dans son Italia Sacra, Ferdinando Ughelli a repris un certain nombre d'éditions de Santoro, y ajoutant quelques autres pièces. Nous n'avons pu en consulter que la seconde édition ${ }^{72}$; on y trouve, au t. VII (dans le cadre du diocèse d'Anglona) les actes suivants : C. 74-75 : Richard le Sénéchal. C. 75-76 : Bohémond II. C. 76-77 : Alexandre et Richard de Chiaromonte. C. 77-78 : Roger II. C. 78-79 : Guillaume I ${ }^{\mathrm{er}}$. C. 82-83 : Frédéric II. C. 99-100 : Ferdinand d'Aragon. Il ajoute trois actes concernant Carbone trouvés ailleurs : c. 79-80 : Tancrède (repris à Rocco Pirro $^{73}$ ). C. 86-90 : acte de mai 1320 provenant des archives de l'évêché d'Anglona. Un sigillion de Bohémond II est encore reproduit dans le t. IX (diocèse de Tarente), c. 128-129: il est conservé à l'Archivio Doria Pamphlili (77) et a été édité par Santoro. L'étape suivante de l'histoire des archives de Carbone ne nous a laissé que des manuscrits. À l'extrême fin du XVII ${ }^{\mathrm{e}}$ siècle, l'abbé général des Basiliens Pietro Menniti a décidé de centraliser ce qui restait des archives des monastères italo-grecs en deux fonds et de les faire cataloguer : les archives des monastères siciliens iront au SS. Salvatore de Messine, celles des établissements du Midi continental à Rome, à S. Basilio de Urbe $e^{74}$.

Menniti s'est particulièrement intéressé au fonds de Carbone — dont une partie a été déposée aux archives de S. Basilio - , composant deux versions (manuscrites) d'un Chronicon Carbonense qui donne des analyses d'une centaine d'actes et en reproduit certains ${ }^{75}$. Pour le composer, il a travaillé à la bibliothèque Pamphili (qu'il désigne sous le sigle $B$. P.), mais on ne sait s'il y a consulté les documents eux-mêmes ou un « Notamento », un catalogue (que Holtzmann n'a pas retrouvé $)^{76}$; il a aussi utilisé le livre de Santoro ; dans sept cas, enfin, il dit avoir utilisé des documents des archives de S. Basilio de Urbe (qui n'existent plus). Il affirme même que les moines de Carbone jouissent d'une franchise pour la mouture du blé « come consta da molte scritture, che si conservano nell'Archivio Basiliano di Roma $»^{77}$.

C'est sans doute au moment de l'occupation française, à la fin du XVIII ${ }^{\mathrm{e}}$ siècle, que ce qui restait des archives de $\mathrm{S}$. Basilio de Urbe a été transporté à l'Archivio Segreto Vaticano ${ }^{78}$ : ainsi s'est constitué le fonds Basiliani, qui ne contient que des pièces modernes ${ }^{79}$; les deux versions du Chronicon Carbonense se trouvent respectivement dans le vol. I ( $\left.f^{\circ} 62 r-128 r\right)^{80}$ et dans le volume XXIII de ce fonds. Il semble enfin que certains actes transcrits par Menniti on été recopiés par d'autres érudits.

En 1991, Gastone Breccia a analysé en détail les actes transmis par Menniti dans le cadre de son étude sur les archives de S. Basilio de Urbe $e^{81}$. Menniti avait donné le texte (ou la traduction) de 20 actes : 6 ont été déjà publiés par Gertrude Robinson, 2 par Holtzmann, 10 par Santoro ; deux restent inédits ${ }^{82}$. Breccia signale encore l'existence de trois actes de Carbone, provenant des archives de $\mathrm{S}$. Basilio et conservés à Grottaferrata ${ }^{83}$, et d'un autre conservé à la Bibliothèque Vaticane $^{84}$; il donne une édition de ces quatre documents ${ }^{85}$, sans doute détachés du fonds de S. Basilio avant son expédition en France à l'époque napoléonienne.

Le XIX ${ }^{\mathrm{e}}$ siècle ne semble pas avoir apporté à l'histoire du fonds d'autres éléments que la traduction italienne du livre de Santoro déjà citée ${ }^{86}$. Le précepte de Frédéric II publié par Ughelli est reproduit par Huillard-Bréholles ${ }^{87}$, un acte de Frédéric II conservé à l'Archivio Doria Pamphili est édité par Winkelmann ${ }^{88}$. D'autre part, à la fin de ce siècle, un érudit calabrais, Rocco Cotroneo, a publié deux actes grecs du fonds de Carbone ${ }^{89}$. Ajoutons encore que Camillo Minieri Riccio publie un acte de $1163^{90}$ concernant le monastère de S. Giacomo de Sarcone, proche de Carbone, venant des «pergamene de' Monasteri soppressi, vol. 2 n. 111 » de l'Archivio di Stato de Naples; si les parchemins des « Monasteri soppressi » ont disparu en 1943, il faudrait s'assurer que les archives napolitaines ne contiennent pas de copies modernes d'actes concernant Carbone ${ }^{91}$. 
Le $\mathrm{XX}^{\mathrm{e}}$ siècle est d'abord représenté par l'édition de Gertrude Robinson. Cette helléniste britannique a donné aux Orientalia Christiana Analecta, en trois livraisons, en 1928, 1929 et 1930, son History and Cartulary of the Greek Monastery of S. Elias and S. Anastasius of Carbone $^{92}$ (introduction historique en I, actes en II et II-2 ; un quatrième volume devait suivre). Elle y retrace d'abord l'histoire de l'abbaye et de ses archives avant de donner une édition de 56 actes grecs (soit l'ensemble de ceux-ci), de 14 actes latins (ou traductions latines d'actes grecs) et de deux actes bilingues : l'un de Guillaume II créant l'archimandritat ( $n^{\circ} \mathrm{XLVI}$ ), l'autre de Constance ( $\mathrm{n}^{\circ} \mathrm{LXVI}$ ), soit au total 72 actes $^{93}$, tous tirés de l'Archivio Doria Pamphili et antérieurs à 1200, alors que Holtzmann estime que, en combinant les diverses sources conservées, on atteindrait une centaine d'actes pour la même période. Ajoutons que les documents latins du bas Moyen Âge (qui contiennent parfois des actes plus anciens insérés) sont également nombreux.

Les reproches qu'on peut faire à l'édition de Gertrude Robinson sont évidents : cette helléniste n'avait manifestement pas reçu de formation en diplomatique ni en histoire ; elle laisse des abréviations non résolues ; ses lectures du latin ne sont pas sûres ; son édition ne répond absolument pas aux critères scientifiques du $\mathrm{XX}^{\mathrm{e}}$ siècle ; enfin, elle n'a pris en compte que les actes conservés à l'Archivio Doria Pamphili. Elle a toutefois eu le mérite de faire sortir de l'oubli ce fonds riche et original, le seul vraiment bilingue qu'on ait conservé, et d'en permettre l'utilisation dans certaines limites. Gertrude Robinson affirme encore avoir vu, grâce au Dott. Barletta de San Chirico [Raparo], une platea du monastère de Carbone, alors en possession d'un avvocato De Nigris, ami de Barletta ${ }^{94}$ : existe-t-elle toujours ? Est-elle visible? Il faut donc impérativement reprendre ses transcriptions avec leur appareil critique et les joindre aux autres sources documentaires venant des archives de Carbone.

En 1956, Walther Holtzmann (Istituto Storico Germanico de Rome) a repris le dossier documentaire de Carbone ; il devait s'en occuper avec André Guillou (École française de Rome) qui aurait pris en charge les actes grecs ${ }^{95}$. Dans l'article qu'il a donné en 1956 à la revue de l'Istituto Storico Germanico, Holtzmann a publié (ou republié) 16 actes, dont 15 provenant de l'Archivio Doria Pamphili (le premier également publié par Santoro et Ughelli) et un ( $\mathrm{n}^{\circ} 3$ ) transcrit par Menniti ; quatre sont conservés dans des copies du bas Moyen Âge ( $\mathrm{n}^{\circ}$ 5, 6, 7 , 13). La qualité de la transcription est évidemment excellente. Holtzmann a notamment réédité la partie latine des actes bilingues de Guillaume II et de Constance déjà publiés par Gertrude Robinson (Holtzmann $n^{\circ} 8=$ Robinson $^{\circ}$ XLVI ; Holtzmann $n^{\circ} 11=$ Robinson $^{\circ}$ LXVI), la partie grecque étant éditée dans le même article par André Guillou.

Il est difficile de savoir ce que Guillou a fait, en dehors de cette transcription. Il a toutefois laissé, dans le volume de Gertrude Robinson qu'il possédait, une nouvelle transcription des actes LXVII et LXVIII, des corrections portant sur les datations et sur les textes et aussi quelques notes : aux $\mathrm{n}^{\text {os }}$ VIII, XI, XVII, LVII, LXVI il renvoie à l'article de Cappelli sur Mormanno ; pour les $n^{\text {os }}$ XXIV et XXVI, il cite Menniti, aux $n^{\text {os }}$ XXVIII, XXIX, XXXI, XXXIV il renvoie à Menniti, Santoro et Ughelli; au n ${ }^{\circ}$ XLVI il signale la reproduction dans l'Archivio paleografico Italiano ${ }^{96}$.

Ajoutons encore quelques études partielles récentes : dans son article sur l'histoire de Mormanno ${ }^{97}$, Biagio Cappelli donne des regestes de 6 actes édités par Gertrude Robinson $\left(\mathrm{n}^{\text {os }}\right.$ VIII, XI, XVII, XX, LVII, LX). Plus récemment les volumes du Codex diplomaticus regni Siciliae consacrés aux actes de Guillaume $\mathrm{I}^{\text {er }}$, de Tancrède et de Constance (ce dernier repris et augmenté dans les $M G H$ ) et le troisième volume publié des actes de Frédéric II dans les $M G H$ republient les actes de ces souverains pour Carbone ${ }^{98}$. Annick Peters-Custot a publié un acte latin du XIV ${ }^{\mathrm{e}}$ siècle ${ }^{99}$. En outre, parmi les documents de Monreale présentés par P. Garufi, comme dans les éditions des Rationes decimarum et des Taxae pro communibus servitiis, ont été publiés quelques documents concernant Carbone ${ }^{100}$. Ne revenons pas sur l'apport de l'article publié par Gastone Breccia en 1991 ; ajoutons qu'il a fourni en 1994 des regestes des documents de Carbone de 1276 à $1473^{101}$ et, avec D. Fugaro, publié un nouvel acte ${ }^{102}$.

Il y a maintenant une vingtaine d'années environ, l'École française a fait photographier les actes de Carbone conservés à l'Archivio Doria Pamphili, sous la conduite d'André Jacob qui, 
avec Jean-Marie Martin, a noté la description de chacune des pièces. C'est en particulier sur ce matériel (malheureusement antérieur à la photographie numérique) que nous comptons nous appuyer pour réunifier et publier les archives du monastère de Carbone.

Les cardinaux et abbés de l'époque moderne (les deux cardinaux Santoro, le cardinal Pamphili, l'abbé Menniti) ont eu le grand mérite de sauver ce qui pouvait l'être de ce fonds, qui est, à l'heure actuelle, le plus important de ceux qui concernent les monastères grecs de l'Italie méridionale ; mais, en le sauvant, ils l'ont fait éclater. Il est temps de le publier intégralement.

\section{Conclusion}

On voit que la tradition des documents du fonds de Carbone est assez complexe, mais a permis la conservation d'un nombre important de chartes. La mise au point globale sur l'état du fonds et ses différentes composantes, que nous avons faite, doit permettre de reconstituer, par ordre chronologique, ce qui reste des archives de l'abbaye ; il suffit, maintenant, de dépouiller systématiquement et de transcrire les différentes parties du fonds : documents conservés à l'Archivio Doria Pamphili, éditions de Santoro (et d'Ughelli), tomes I et XXIII du fonds «Basiliani » de l'Archivio Segreto Vaticano, en s'appuyant sur les éditions de Gertrude Robinson et de Walther Holtzmann et sur les notes d'André Guillou et en y ajoutant les documents de Grottaferrata et de la Bibliothèque Vaticane publiés par Gastone Breccia. On trouvera, évidemment, un certain nombre de doublons, donnant parfois un texte grec et sa traduction latine, ou deux versions légèrement différentes d'un même acte. Il faudra publier à la fois les originaux grecs et leurs traductions latines (sans parler, évidemment, des actes originellement bilingues); on devra aussi prendre en compte les simples régestes, même s'ils doublent un original conservé. Il faudra traiter à part les copies insérées dans des actes tardifs. On doit évidemment maintenir le caractère bilingue du fonds, c'est-à-dire publier, en ordre chronologique, à la fois les actes grecs et les latins, ce qui pose un petit problème technique, car on n'édite pas de la même façon les uns et les autres ; nous les traiterons donc ensemble, mais un peu différemment : pour les actes grecs, nous suivrons le modèle de l'édition des actes de l'Athos ; les actes latins bénéficieront d'une analyse nettement plus courte, dans la tradition des publications françaises et italiennes les plus récentes. Les archives contiennent, apparemment, quelques actes du XVI ${ }^{\mathrm{e}}$ siècle, mais aucun postérieur : il semble normal de publier l'ensemble du fonds, qui reste essentiellement médiéval. On doit, en première approximation, arriver à un total d'environ 150 actes au moins. On y ajoutera la platea signalée par Gertrude Robinson, si elle est encore trouvable. Si on repère des actes concernant Carbone issus de fonds autres que celui de l'abbaye (les additions d'Ughelli, d'autres éventuellement déposés à Naples), on les éditera en appendice.

On ne s'attend pas à trouver beaucoup d'actes faux ou falsifiés : les traductions latines d'actes grecs faites aux $\mathrm{XVI}^{\mathrm{e}}$ et $\mathrm{XVII}{ }^{\mathrm{e}}$ siècles n'ont, a priori, pas les mêmes motivations intéressées pour changer le contenu des actes que celles qui ont été faites, ailleurs, au Moyen Âge. Les archives concernant la Basilicate médiévale sont, au total, très rares; le fonds de Carbone est, de loin, le plus important de la région. Il présente l'avantage de permettre de suivre un cas concret et complexe d'acculturation - de la culture latine à la culture grecque au début de l'époque normande, dans l'autre sens ensuite.

Mais les actes de Carbone peuvent éclairer bien d'autres phénomènes : la vie et la fortune d'un grand monastère grec, devenu centre d'un archimandritat ; l'économie, le peuplement et la structure sociale de cette région peu documentée. En ce sens, la publication globale des archives de Carbone ne constituera pas un simple apport documentaire à l'hisoire médiévale de l'Italie méridionale : elle comblera une importante lacune. 


\section{Annexe}

\section{Carte monastique de la Basilicate (Peters-Custot 2009 a, p. 601)}

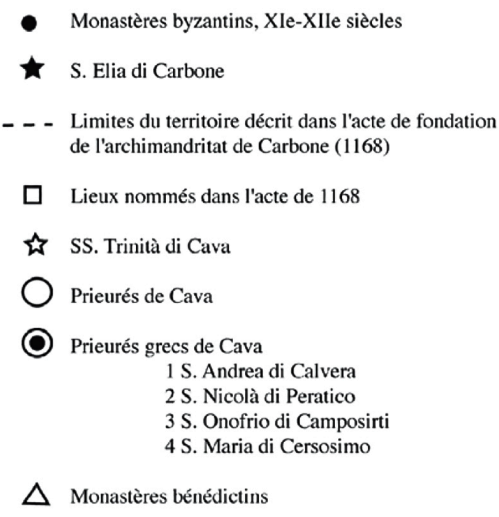

- Monastères byzantins, XIe-XIle siècles

- S. Elia di Carbone

- - - Limites du territoire décrit dans l'acte de fondation de l'archimandritat de Carbone (1168)

$\square \quad$ Lieux nommés dans l'acte de 1168

SS. Trinità di Cava

Prieurés de Cava

- Prieurés grecs de Cava

$1 \mathrm{~S}$. Andrea di Calvera

$2 \mathrm{~S}$. Nicolà di Peratico

3 S. Onofrio di Camposirti

4 S. Maria di Cersosimo

$\triangle$ Monastères bénédictins

(1172) Terminus ante quem de la fondation

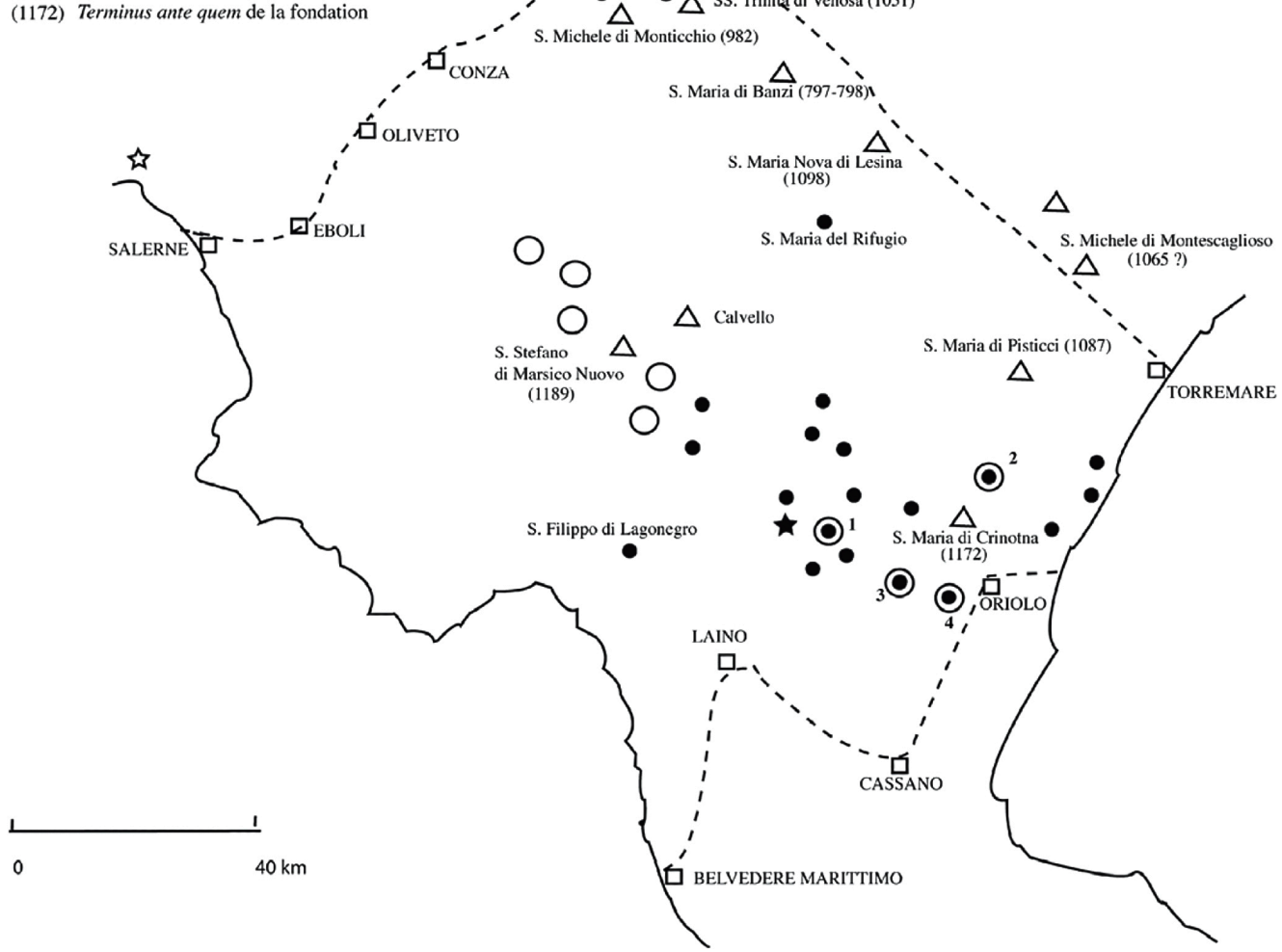

\section{Bibliographie}

Acconcia Longo 1994 : A. Acconcia Longo, Santi monaci italogreci alle origini del monastero di Carbone, dans Fonseca, Lerra (éd.) 1994, p. 47-60.

API 1954 : Archivio paleografico italiano, vol. XIV, fasc. 61, Rome, 1954.

Batiffol 1888 : P. Batiffol, Ungedruckte Papst- und Kaiserurkunden aus basilianischen Archiven, dans Römische Quartalschrift für christliche Alterthumskunde und für Kirchengeschichte, 2, 1888, p. 36-63.

Batiffol 1889 a : P. Batiffol, La Vaticane depuis Paul III, dans Revue des Questions Historiques, 45 (n. s. 1), 1889, p. 177-218.

Batiffol 1889 b : P. Batiffol (sans titre), dans Bulletin de la Société Nationale des Antiquaires de France, 1889 , p. 108-113.

Batiffol 1891 : P. Batiffol, L'abbaye de Rossano. Contribution à l'histoire de la Vaticane, Paris, 1891.

Becker (éd.) 2013 : J. Becker, Edizione critica dei documenti greci e latini di Ruggero I, conte della Calabria e della Sicilia (1080-1101), Roma, 2013 (Ricerche dell'Istituto Storico Germanico di Roma).

Borghese 2009 : G. L. Borghese, Carlo I d'Angio e il Mediterraneo. Politica, diplomazia e commercio internazionale prima dei Vespri, Rome, 2009 (Collection de l'École française de Rome, 411). 
Bougard 2015 : Liutprand de Crémone, Euvres. Présentation, traduction et commentaire par F. Bougard, Paris, 2015 (Sources d'Histoire médiévale, 41).

Breccia 1991 : G. Breccia, Archivum Basilianum. Pietro Menniti e il destino degli archivi monastici italogreci, dans Quellen und Forschungen aus Italienischen Archiven und Bibliotheken, 71, 1991, p. 14-105.

Breccia 1994 : G. Breccia, Il monastero di Carbone dalla conquista angioina alla commenda, dans Fonseca, Lerra (éd.) 1994, p. 131-147.

Breccia 1997 : G. Breccia, Scritture greche di età bizantina e normanna nelle pergamene del monastero di S. Elia di Carbone, dans Archivio storico per la Calabria e la Lucania, 64, 1997, p. 33-89.

Breccia, Fugaro 1994 : G. Breccia, D. Fugaro, Scritture latine di età normanna nei documenti del monastero di S. Elia di Carbone, dans Archivio storico per la Calabria e la Lucania, 61, 1994, p. 5-36.

Bresc 1986 : H. Bresc, Un monde méditerranéen. Économie et société en Sicile, 1300-1450, Rome, 1986 (BEFAR, 262).

Brodbeck 2010 : S. Brodbeck, Les saints de la cathédrale de Monreale en Sicile, Rome, 2010 (Collection de l'École française de Rome, 432).

Brodbeck, Martin, Peters-Custot, Prigent (éd.) 2015 : S. Brodbeck, J.-M. Martin, A. Peters-Custot, V. Prigent, L'héritage byzantin en Italie (VIII $-X I I^{e}$ s. ). III. Décor monumental, objets, tradition textuelle, Rome, 2015 (Collection de l'École francaise de Rome, 510).

Burgarella 2002 : F. Burgarella, Presenze greche a Roma : aspetti culturali e religiosi, dans Roma fra Oriente e Occidente. Atti delle Settimane di studio del centro italiano di studi sull'alto Medioevo, 19-24 aprile 2001, 2 vol., Spolète, 2002, II, p. 943-988.

Cappelli 1941-1942 : B. Cappelli, Note e documenti per la storia di Mormanno, dans Archivio Storico per la Calabria e la Lucania, 11, 1941, p. 161-181 et 12, 1942, p. 27-42

Cherubini 1990 : P. Cherubini, Le pergamene di S. Nicola di Gallucanta (IX-XII), Altavilla Salentina, 1990 (Fonti per la storia del Mezzogiorno, 9).

Chiesa (éd.) 1898 : Liutprandi Cremonensis Opera omnia, éd. P. Chiesa, Turnhout, 1998 (Corpus Christianorum. Continuatio medievalis, 156).

Cotroneo 1898 : R. Cotroneo, Nuove pergamene greche del secolo XII, dans Rivista storica Calabrese, 1898, p. 388-393.

Cotroneo 1902 : [R. Cotroneo], Pergamene greche del secolo XIII, dans Rivista storica Calabrese, 10, 1902, p. 35-43.

Cozza Luzi (éd.) 1893 : G. Cozza-Luzi (éd.), Historia et Laudes SS. Sabae et Macarii juniorum e Sicilia, auctore Oreste, patriarcha Hierosolymitano, Rome 1893.

Ditchfield 2007 : Ph. Ditchfield, La culture matérielle médiévale. L'Italie méridionale byzantine et normande, Rome, 2007 (Collection de l'école française de Rome, 373).

Enzensberger 1973 : H. Enzensberger, Der « Ordo Sancti Basilii », eine monastische Gliederung der römischen Kirche (12.-16. Jahrhundert), dans La Chiesa greca in Italia dall'VIII al XVI secolo, Atti del convegno storico interecclesiale, (Bari, 30 aprile-4 maggio 1969), Padoue, 1973 (Italia Sacra, studi e documenti di storia ecclesiastica, 22), p. 1139-1151.

Enzensberger (éd.) 1996 : Guillelmi I. Regis Diplomata, éd. H. Enzensberger, Cologne-Vienne, 1996 (Codex Diplomaticus Regni Siciliae. Series I, III).

Falkenhausen 1968 : V. von Falkenhausen, Taranto in epoca bizantina, dans Studi medioevali, Ser. III, 9, 1968-1, p. 133-166.

Falkenhausen 1983 : V. von Falkenhausen, Patrimonio e politica patrimoniale dei monasteri greci nella Sicilia normanna-sveva, dans Basilio di Cesarea. La sua età, la sua opera e il basilianesimo in Sicilia. Atti del Congresso internazionale, Messina, 3-6 decembre 1979, 2 vol., Messine, 1983, II, p. 777-790.

Falkenhausen 1994 a : V. von Falkenhausen, L'archimandritato del S. Salvatore in lingua Phari di Messina e il monachesimo italo-greco nel regno normanno-svevo (secoli XI-XIII), dans Messina. Il ritorno della memoria. Catalogue de l'exposition de Messine (Palazzo Zanca), $1^{\text {er }}$ mars-28 avril 1994, Palerme, 1994, p. 44-50.

Falkenhausen 1994 b: V. von Falkenhausen, Il monastero dei SS. Anastasio ed Elia di Carbone in epoca bizantina e normanna, dans Fonseca, Lerra 1994, p. 61-87.

Falkenhausen 1998 : V. von Falkenhausen, Gregor von Burtscheid und das griechische Mönchtum in Kalabrien , dans Römische Quartalschrift, 93, 1998, p. 215-250. 
Falkenhausen 2014 a : V. von Falkenhausen, Il monachesimo greco nel Lazio medievale, dans G. Bordi et al. (a cura di), L'officina dello sguardo. Scritti in onore di Maria Andaloro, I. I luoghi dell'arte, Rome, 2014, p. 305-314.

Falkenhausen 2014 b : V. von Falkenhausen, La documentazione greca della Badia di Cava e il monachesimo italo-greco nei secoli XI-XII, dans Riforma della Chiesa, esperienze monastiche e poteri locali. La Badia di Cava nei secoli XI-XII. Atti del Convegno internazionale di studi (Badia di Cava, 15-17 settembre 2011), éd. M. Galante, G. Vitolo, G. Z. Zanichelli, Florence, 2014 (Millennio Medievale, 99), p. 161-181.

Fonseca, Lerra (éd.) 1994 : C. D. Fonseca, A. Lerra (éd.), Il monastero di S. Elia di Carbone e il suo territorio dal Medioevo all'Età moderna. Nel millenario della morte di S. Luca Abate. Atti del Convegno internazionale di studio Potenza-Carbone 26-27 giugno 1992, Galatina, 1994 (Università degli studi della Basilicata - Potenza. Atti e Memorie, 16).

Garufi 1902 : P. Garufi, Catalogo illustrato del tabulario di S. Maria Nuova in Monreale, Palerme, 1902 (Documenti per servire alla storia di Sicilia, I, 19).

Grévin 2008 : B. Grévin, Rhétorique du pouvoir médiéval. Les Lettres de Pierre de la Vigne et la formation du langage politique européen (XIII - XV siècle), Rome, 2008 (BEFAR, 338).

Guillou (éd.) 1967 : Saint-Nicolas de Donnoso (1031-1060-1061), éd. A. Guillou, Cité du Vatican, 1967 (Corpus des actes grecs d'Italie et de Sicile, I).

Guillou (éd.) 1972 : La Théotokos de Hagia-Agathè (Oppido) (1050-1064/1065), éd. A. Guillou, Cité du Vatican, 1972 (Corpus des Actes Grecs d'Italie et de Sicile, III)

Guillou 1996 : A. Guillou, Recueil des inscriptions grecques médiévales d'Italie, Rome, 1996 (Collection de l'École française de Rome, 222)

Guillou (éd.) 2009 : Les actes grecs des fonds Aldobrandini et Miraglia (XI'-XIII ${ }^{e}$ s.), éd. A. Guillou, Cité du Vatican, 2009 (Corpus des actes grecs d'Italie et de Sicile, VI)

Hoberg 1949 : H. Hoberg, Taxae pro communibus servitiis ex libris obligationum ab anno 1295 usque ad annum 1455 confectis, Cité du Vatican, 1949 (Studi e testi, 144).

Holtzmann 1956 : W. Holtzmann, Papst-, Kaiser- und Normannenurkunden aus Unteritalien. V. S. Elia di Carbone, dans Quellen und Forschungen aus italienischen Archiven und Bibliotheken, 36, 1956, p. 34-85.

Huillard-Bréholles 1859-1861 : J.-L.-A. Huillard-Bréholles, Historia diplomatica Friderici secundi, Paris 1859-1861, 12 vol.

Jacob 1980 : A. Jacob, Culture grecque et manuscrits en Terre d'Otrante, dans Atti del III ${ }^{\circ}$ Congresso internazionale di Studi Salenti e del $I^{\circ}$ Congresso storico di Terra d'Otranto (Lecce, 22-25 octobre 1976), Lecce, 1980, p. 53-77.

Jacob 1982 : A. Jacob, Sergio Stiso de Zollino et Nicolas Petreo de Curzola. À propos d'une lettre du Vaticanus gr. 1019, dans Bisanzio e l'Italia . Raccolta di Studi in memoria di Agostino Pertusi, Milan, 1982, p. 154-168.

Jacob 1983 : A. Jacob, Un nouveau manuscrit des Hymnes Orphiques et son copiste, François Cavoti de Soleto, dans L'Antiquité classique, 52, 1983, p. 246-254.

Jacob 1991 : A. Jacob, Les annales d'une famille sacerdotale grecque de Galatina dans l'Ambrosianus C 7 Sup. et la peste en Terre d'Otrante à la fin du Moyen Âge, dans Bollettino storico di Terra d'Otranto, 1, 1991, p. 23-51.

Jacob 1992 : A. Jacob, Le nom de famille du dernier copiste grec de Gallipoli, dans Bollettino storico di Terra d'Otranto, 2, 1992, p. 77-83.

Jacob 1995 : A. Jacob, Une date précise pour l'euchologe de Carbone : 1194-1195, dans Archivio storico per la Calabria e la Lucania, 62, 1995, p. 97-114.

Jacob, Martin, Noyé (éd.) 2006 : A. Jacob, J.-M. Martin et G. Noyé (dir.), Histoire et culture dans l'Italie byzantine, Rome, 2006 (Collection de l'École française de Rome, 363).

Koch (éd.) 2010 : M. Koch (éd.), unter Mitwirkung von K. Höflinger, J. Spiegel und Ch. Friedl, Die Urkunden Friedrichs II. 1218-1220, Hanovre, 2010 (MGH, Diplomata, XIV-3).

Kölzer (éd.) 1983 : Th. Kölzer (éd.), Constantiae imperatricis et reginae Siciliae diplomata (1195-1198), Cologne-Vienne, 1983 (Codex diplomaticus regni Siciliae, II, I-2).

Kölzer (éd.) 1990 : Th. Kölzer (éd.), Constantiae imperatricis diplomata, Hanovre, 1990 (MGH, Diplomata, XI-3). 
Korolevskij 1932 : C. Korolevskij, Basiliens italo-grecs et espagnols, dans Dictionnaire d'histoire et de géographie ecclésiastiques, VI, Paris, 1932, c. 1180-1236.

Laurent-Guillou 1960 : M.-H. Laurent et A. Guillou, le 'Liber visitationis' d'Athanase Chalkéopoulos (1457-1458). Contribution à l'histoire du monachisme grec en Italie méridionale, Cité du Vatican, 1960 (Studi e testi, 206).

Lauwers 2013 : M. Lauwers, Réforme, romanisation, colonisation ? Les moines de Saint-Victor de Marseille en Sardaigne (seconde moitié XI'-première moitié XII ${ }^{\mathrm{e}}$ siècle), dans Cahiers de Fanjeaux, 48, La réforme " grégorienne » dans le midi (milieu XI ${ }^{e}$-début XIII ${ }^{e}$ siècle), 2013, p. 257-310.

L'État angevin 1998 : L'Etat angevin : pouvoir, culture et société entre XIII et XIV siècle, Actes du colloque international, organisé par l'American Academy in Rome, l'École française de Rome, l'Istituto storico italiano per il Medio Evo, Rome-Naples, 7-11 novembre 1995, Rome, 1998 (Collection de l'École française de Rome, 245 ; Nuovi Studi storici, 45).

Marchionibus 2004 : M. R. Marchionibus, Il Cilento bizantino. Monastero di S. Maria de Pactano, Salerne, 2004.

Martin 1985-1986 : J.-M. Martin, Une origine calabraise pour la Grecia salentine ? , dans Rivista di Studi Bizantini e Neoellenici, 22-23, 1985-1986, p. 51-63, rééd. dans Martin 2014, p. 39-47.

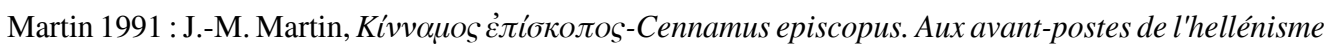
sud-italien vers l'an mil, dans Rivista di Studi Bizantini e Neoellenici, n.s. 27, 1990, Rome, 1991, p. 89-99, rééd. dans Martin 2014, p. 67-74.

Martin 1993 : J.-M. Martin, La Pouille du VI au XII siècle, Rome, 1993 (Collection de l'École français de Rome, 179).

Martin 2005 : J.-M. Martin, Guerre, accords et frontières en Italie méridionale pendant le haut Moyen Âge. Pacta de Liburia, Divisio principatus Beneventani et autres textes, Rome, 2005 (Sources et documents d'histoire du Moyen Âge publiés par l'École française de Rome, 7).

Martin 2006 : Les institutions liées à la conquête. Le duché, dans R. Licinio et F. Violante (dir.), I caratteri originali della conquista normanna. Diversità e identità nel Mezzogiorno (1030-1130). Atti delle sedicesime Giornate normanno-sveve (Bari, 5-8 ottobre 2004), Bari, 2006, p. 305-333, rééd. dans Martin 2014, p. 481-501.

Martin 2014 : Byzance et l'Italie méridionale, Paris, 2014 (Association des Amis du Centre d'histoire et civilisation de Byzance, Bilans de recherche, 9).

Martin, Peters-Custot, Prigent (éd.) 2011 : J.-M. Martin, A. Peters-Custot, V. Prigent, L'héritage byzantin en Italie (VIII'-XII ${ }^{e}$ s.). I. La fabrique documentaire, Rome, 2011 (Collection de l'École française de Rome, 449).

Martin, Peters-Custot, Prigent (éd.) 2012 : J.-M. Martin, A. Peters-Custot, V. Prigent, L'héritage byzantin en Italie (VIII ${ }^{e}-X I^{e}$ s.). II. Les cadres juridiques et sociaux et les institutions publiques, Rome, 2012 (Collection de l'École française de Rome, 461).

Martin et al. (éd.) 2015 : J.-M. Martin, P. Chastang, E. Cuozzo, L. Feller, G. Orofino, A. Thomas, M. Villani (éd.), Registrum Petri Diaconi (Montecassino, archivio dell'abbazia, Reg. 3). Edizione e commento, Rome, 2015, 4 vol. (Sources et documents publiés par l'École française de Rome, 4 ; Fonti per la storia dell'Italia medievale. Antiquitates, 45).

Mercati 1935 : G. Mercati, Per la storia dei manoscritti greci di Genova di varie badie basiliane d'Italia e di Patmo, Cité du Vatican, 1935 (Studi e testi, 68).

Mercati, Giannelli, Guillou (éd.) 1980 : Saint-Jean-Théristès (1054-1264), éd. S. G. Mercati, C. Giannelli, A. Guillou, Cité du Vatican, 1980 (Corpus des actes grecs d'Italie et de Sicile, V).

Minieri Riccio 1878 : C. Minieri Riccio, Saggio di Codice diplomatico formato sulle antiche scritture dell'Archivio di Stato di Napoli, I, Naples, 1878.

Minisci 1952 : T. Minisci, Il fondo «Basiliani » dell'archivio segreto vaticano, dans Bollettino della Badia Greca di Grottaferrata, n. s. 6, 1952, p. 65-85.

Nef 2011 : A. Nef, Conquérir et gouverner la Sicile islamique aux XI et XII siècles, Rome, 2011 (BEFAR, 346).

Patlagean 2008 : E. Patlagean, Les hommes (anthrôpoi) dans les documents grecs du Mezzogiorno normand, dans Puer Apuliae. Mélanges offerts à Jean-Marie Martin, éd. E. Cuozzo, V. Déroche, A. Peters-Custot et V. Prigent, Paris, 2008 (Centre de recherche d'histoire et de civilisation de Byzance. Monographies, 30), II, p. 529-536. 
Peters-Custot 2002 : A. Peters-Custot, Le monastère de Carbone au début du XIV siècle, dans MEFRM, 114-2, 2002, p. 1045-1066.

Peters-Custot 2006 : A. Peters-Custot, Les communautés grecques de Basilicate à l'époque byzantine, dans Jacob, Martin, Noyé 2006, p. 559-587.

Peters-Custot 2009 a : A. Peters-Custot, Les Grecs de l'Italie méridionale post-byzantine. Une acculturation en douceur (IX $-X I V^{e}$ s.), Rome, 2009 (Collection de l'École française de Rome, 420).

Peters-Custot 2009 b : A. Peters-Custot, L'identité d'une communauté minoritaire au Moyen Âge. La population grecque de la principauté lombarde de Salerne, IX'-XII siècles, dans MEFRM, 121-1, 2009, p. 83-97.

Peters-Custot 2014 : A. Peters-Custot, Bruno en Calabre. Histoire d'une fondation monastique dans l'Italie normande : S. Maria de Turri et S. Stefano del Bosco, Rome, 2014 (Collection de l'École française de Rome, 489).

Peters-Custot 2015 : A. Peters-Custot, ... Et saint Basile en Occident, dans S. Excoffon, D.-O. Hurel et A. Peters-Custot (dir.), Interactions, emprunts, confrontations chez les religieux (Antiquité tardive-fin du XIX ${ }^{e}$ siècle). Actes du VIII Colloque international du CERCOR. Célébration du trentenaire (1982-2012), Saint-Etienne, 24-26 octobre 2012, Saint-Etienne, 2015, p. 93-112.

Peters-Custot s. p. (a) : A. Peters-Custot, Stratégies épistolaires et réforme grégorienne : le cas de l'Italie méridionale normande, dans Epistolaire politique IV : Lettres et réseaux. Actes du colloque international organisé par Laurent Vissière et Bruno Dumézil, Université de Sao Paulo, 15-17 avril 2014, sous presse.

Peters-Custot s. p. (b) : A. Peters-Custot, Bessarion's monastic rule, the modalities of the text's redaction, and its impact on the Italian-Greek "Basilian » monasteries, dans Bessarion's treasure: Editing, translating and interpreting Bessarion's literary heritage. International Conference, Venice, 4 and 5 April 2014, sous presse.

Peters-Custot s. p. (c) : A. Peters-Custot, Qu'est-ce qu'être "grec » dans l'Italie méridionale médiévale? A propos d'une « identité » polysémique et en perpétuel mouvement, dans Identité religieuse et minorités, de l'Antiquité au XVIII' siècle. Colloque international organisé à l'Université d'Angers, 12-13 juin 2014, sous presse.

Peters-Custot s. p. (d) : A. Peters-Custot, Clairvaux et l'ordre cistercien dans un espace en marge de la chrétienté romaine : le royaume de Sicile aux époques normande et souabe, dans Le temps long de Clairvaux. Nouvelles recherches, nouvelles perspectives (XII ${ }^{e}-X X I^{e}$ siècle), Colloque internaitonal Troyes-Clairvaux, 16-18 juin 2015, sous presse.

Petta 1971 : M. Petta, L'originale dell'inventario dei libri del monastero di S. Elia di Carbone, dans Bollettino della Badia greca di Grottaferrata, 25, 1971, p. 62-64.

Petta 1972 : M. Petta, Codici del Monastero di S. Elia di Carbone conservati nella biblioteca dell'Abbazia di Grottaferrata, dans Vetera Christianorum, 9, 1972, p. 151-171

Petta 1994 : M. Petta, I manoscritti greci di S. Elia di Carbone, dans Fonseca-Lerra (éd.) 1994, p. 97-110.

Pirro 1733 : R. Pirro, Sicilia Sacra, Palerme, 1733.

Poncet 2004 : O. Poncet, Innocenzo X, dans Dizionario biografico degli Italiani, 62, Rome, 2004, p. $466-478$.

Robinson 1928-1930 : G. Robinson, History and Cartulary of the Greek Monastery of S. Elias and S. Anastasius of Carbone. I. History, Orientalia Christiana, vol. XI.5, num. 44, Rome, 1928. II. Cartulary, Orientalia Christiana, vol. XV.2, num. 53, Rome, 1929, II-ii Cartulary, Orientalia Christiana, vol. XIX.1, num. 62, Rome, 1930

Robinson 1930 : G. Robinson, Some Cave Chapels of Southern Italy, dans Journal of Hellenic Studies, 50-2, 1930, p. 186-209.

Rognoni (éd.) 2004 : C. Rognoni, Les actes privés grecs de l'Archivo Ducal de Medinaceli (Tolède), I., Les monastères de Saint-Pancrace de Briatico, de San Filippo de Bojôannès, et de S. Nicola-des-Drosi (Calabre, $X I^{e}-X I I^{e}$ siècles), Paris, 2004 .

Rognoni 2011 : C. Rognoni, Les actes privés grecs de l'Archivo Ducal de Medinaceli (Tolède), II., La vallée du Tuccio (Calabre, XII ${ }^{e}$-XIII' siècles), Paris, 2011.

Sansterre 1980 : J.-M. Sansterre, Les moines grecs et orientaux à Rome aux époques byzantine et carolingienne (milieu du VI $I^{e}$ s.-fin du IX $X^{e}$ s.), Bruxelles, 1980, 2 vol.

Santoro 1601 : Pauli Æmilii Sanctorii Casertani Historia monasterii Carbonensis ordinis sancti Basilii, Romae, Apud Guillielmum Faciottum, 1601.

Santoro 1831 : P. E. Santoro, Storia del monastero di Carbone, trad. M. Spena, Naples, 1831. 
Scaduto 1982 : M. Scaduto, Il monachesimo basiliano nella Sicilia medievale. Rinascita e decadenza, sec. XI-XIV, Rome, 1982.

Trinchera (éd.) 1865 : F. Trinchera, Syllabus Graecarum Membranarum..., Naples, 1865

Ughelli-Coleti 1717-1722 : F. Ughelli et N. Coleti, Italia Sacra, Venise, 1717-1722, 10 vol..

Vaccari 1925 : A. Vaccari, La Grecia nell'Italia meridionale. Studi letterari e biografici, Orientalia Christiana, vol. III.3, num. 13, Rome, 1925, p. 273-328.

Vendola 1939 : D. Vendola, Rationes decimarum Italiae nei secoli XIII e XIV. Puglia-Lucania-Calabria, Cité du Vatican, 1939 (Studi e testi, 84).

Vitolo 1982 : G. Vitolo, La latinizzazione dei monasteri italo-greci del Mezzogiorno medievale, l'esempio di S. Nicola di Gallocanta presso Salerno, dans Benedictina, 29, 1982, p. 437-450, rééd. dans S. Leone et G. Vitolo, Minima Cavensia. Studi in margine al IX volume del Codex Diplomaticus Cavensis, Salerne, 1983 (Iter Campanum, 1), p. 75-92.

Winkelmann 1880-1885 : E. Winkelmann, Acta imperii inedita seculi XIII, Innsbruck, 1880-1885, 2 vol.

Zielinski (éd.) 1982 : H. Zielinski (éd.), Tancredi et Willelmi III regum diplomata, Cologne-Graz, 1982 (Codex diplomaticus regni Siciliae, I, v).

\section{Notes}

1 Bresc 1986.

2 Martin 1993. On ajoutera cette publication plus récente : Jacob, Martin, Noyé (éd.) 2006.

3 Borghese 2009. Sur l'État angevin voir également L'Etat angevin 1998.

4 Brodbeck 2010.

5 Ditchfield 2007.

6 Grévin 2008.

7 Nef 2011.

8 Peters-Custot 2009 a ; Peters-Custot 2014.

9 On espère disposer prochainement de la publication de la thèse de Vivien Prigent sur la Sicile byzantine. Pour patienter, on retiendra la parution en cours des actes des cinq colloques qui se sont déroulés à Rome, avec la forte implication de l'École française de Rome, de 2008 à 2011 sur le thème de L'héritage byzantin en Italie, VIII'-XII siècles; les volumes suivants sont parus : Martin, Peters-Custot, Prigent (éd.) 2011 ; Martin, Peters-Custot, Prigent (éd.) 2012; Brodbeck, Martin, Peters-Custot, Prigent (éd.) 2015.

10 Mentionnons le volume épigraphique de Guillou 1996. On se réfère toutefois surtout aux collections « Sources et documents d'histoire du Moyen Âge » et « Sources et documents », dans lesquelles ont paru divers volumes voués aux documents de l'Italie méridionale, notamment Martin et al. (éd.) 2015.

11 « Rompant avec une historiographie ancienne (...), les travaux réalisés au cours des dernières années ont qualifié de 'grégorien' le processus de transformation de l'institution ecclésiale engagé dans l'Occident de la seconde moitié du XI siècle et montré son impact sur les modèles culturels et les structures sociales, en s'attachant tout particulièrement aux réalités locales et aux configurations régionales : la réforme imposée par la papauté y est dès lors envisagée comme un nœud de pratiques sociales, fait d'affrontements et de ruptures, mais aussi de négociations et de compromis au sein de l'aristocratie » (Lauwers 2013, p. 257).

12 Voir Peters-Custot, s. p. (a) ; Peters-Custot, s. p. (d).

13 L'étude fondamentale sur le monachisme oriental à Rome au haut Moyen Âge reste celle de Sansterre 1980. On pourra lui adjoindre Burgarella 2002.

14 Sur les populations hellénophones nouvellement venues à Salerne et dans le Latium, on renvoie aux notes suivantes. Concernant Tarente, le monopole de la production d'actes grecs au haut Moyen Âge a pu faire penser que la ville était majoritairement peuplée d'hellénophones, option suivie par Vera von Falkenhausen (Falkenhausen 1968) mais qui a été contestée, en mettant en avant le trompte-l'œil de la conservation documentaire (Martin 1993, p. 512) ; voir encore Martin 1991.

15 A ce sujet, on renvoie à Martin 1985-1986, et à Peters-Custot 2009 b. 
16 A côté du monastère de Sainte-Marie de Grottaferrata, célèbre fondation de Nil de Rossano, tout près de Rome, et sur laquelle la bibliographie est désormais infinie, on mentionnera des fondations grecques plus menues dans le Latium : voir, à ce sujet Falkenhausen $2014 \mathrm{a}$.

17 Le principal représentant du monachisme grec de la principauté de Salerne est Saint-Nicolas de Gallocanta, dont les actes ont été édités : Cherubini 1990. Sur ce monastère voir Vitolo 1982. Sur les Grecs de Salerne, qui jouissaient d'un «juge des Grecs » ainsi que d'un « chorévêque », voir Peters-Custot 2009 b.

18 Falkenhausen 2014 b ; Marchionibus 2004, en particulier p. 43-53.

19 On fait mention ici du monastère de Burtscheid, fondé par Grégoire de Cassano, parti en Germanie à la suite de la cour de l'impératrice Théophanô, épouse d'Otton II. Voir Falkenhausen 1998.

20 Il manque une étude de synthèse sur le monachisme grec dans la Sicile des Hauteville, et sur son rôle dans la rechristianisation de l'île. On se reportera donc pour l'instant au classique ouvrage suivant : Scaduto 1982, en attendant la réalisation du doctorat d'histoire que Dmitri Royer, doctorant de l'université de Nantes, prépare sur la question.

21 Les études d'André Jacob sur le Salento ont démontré que la culture grecque était largement répandue dans le milieu des prêtres séculiers, et non uniquement dans celui des monastères italo-grecs. Voir Jacob 1980 ; Jacob 1982 ; Jacob 1983 ; Jacob 1991 ; Jacob 1992.

22 Peters-Custot 2009 a, p. 266-306, 510-542, et 574-576.

23 Sur cette règle de Bessarion pour le monachisme italo-grec, voir en premier lieu l'introduction au Liber Visitationis d'Athanase Chalkéopoulos, dans Laurent-Guillou 1960. A. Peters-Custot prépare une réédition du texte accompagnée de commentaires. Voir A. PetersCustot s. p. (b).

24 Sur l'ordre basilien, créé pour le monachisme italo-grec au $\mathrm{XV}^{\mathrm{e}}$ siècle, mais qui connut des ramifications mutiples à l'époque moderne, dans les Balkans, en Lituanie, au Liban, et même en Espagne, la synthèse de Cyril Korolevskij (Korolevskij 1932) reste très utile. Une journée d'études a été organisée par Olivier Delouis et Annick Peters-Custot, le 3 novembre 2015 : « De Basile aux Basiliens. La postérité monastique d'un Père grec en Orient et en Occident, du Moyen Âge à l'époque contemporaine » http://www.efrome.it/fileadmin/res/ PDF_Flyers_Affiches_Programmes/Moyen_Age/3Prog_Basile_aux Basiliens.pdf. Sur les prémices de la création de la « règle », puis de « l'ordre » de saint Basile, voir Peters-Custot 2015.

25 Font exception les actes grecs des évêchés méridionaux : ainsi Guillou (éd.) 1972 ; de même, les actes grecs conservés dans les archives d'abbayes latines, par exemple ceux de Cava ou du Mont-Cassin, ou encore Guillou (éd.) 2009 ; ainsi que les actes grecs des souverains Hauteville, qui n'étaient pas tous conservés dans des fonds monastiques, et qui côtoient des documents latins, arabes, voire multilingues, l'essentiel ayant été produit avant le règne de Guillaume II. Pour le comte Roger I ${ }^{\text {er }}$, par exemple, on se reportera à Becker (éd.) 2013. Pour Guillaume I ${ }^{\text {er }}$, voir Enzensberger (éd.) 1996.

26 Voir notamment, pour les archives monastiques, Guillou (éd.) 1967 ; Mercati, Giannelli, Guillou (éd.) 1980.

27 Rognoni 2004 ; Rognoni 2011.

28 Trinchera (éd.) 1865.

29 Falkenhausen 2014 b.

30 Robinson 1928-1930. Gertrude Robinson a disparu en Italie peu après cette publication, ce qui ne manque pas d'alimenter le mystère sur l'intérêt curieux qu'elle a nourri à l'égard des actes italo-grecs médiévaux, dont cette édition est le produit. Elle est aussi l'auteur d'un article contemporain à cette publication, et portant sur les églises rupestres d'Italie du Sud : Robinson 1930, p. 186-209.

31 Robinson 1928-1930, doc. VIII-59 (1058-1059).

32 Le Latinianon est en effet une circonscription administrative ancienne, mentionnée comme telle dans la Divisio de 848 (ou 849) : Le texte, intitulé Praeceptum concessionis sive capitulare, est édité dans Martin 2005, p. 201-217.

33 Peters-Custot 2006. 
34 Pour ce qui est du droit byzantin, légèrement teinté d'altérations lombardes, voir la mise au point dans Peters-Custot 2009 a, p. 146-150 ; Peters-Custot s. p. (c). En ce qui concerne les rapports sociaux et les statuts de paysans, soulignons qu'on a longtemps estimé que le terme de parèque ne connaissait pas d'attestation antérieure à l'établissement de la monarchie (1130). Or, justement, un acte grec de Carbone contient une mention plus précoce, datée de 1108 : Robinson 1928-1930, $\mathrm{n}^{\circ}$ XVII. La donation que Trotta, dame de Myromanna (Mormanno),

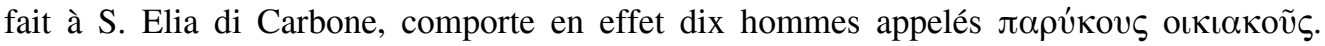
Généralement, l'usage du terme de parèques (plutôt que celui, plus simple, d' " hommes »), est associé à une préciosité de notaire grec jaloux de son lexique élevé et byzantinisant. C'est du moins l'interprétation avancée par Evelyne Patlagean (Patlagean 2008). Or, l'occurrence du document daté de 1108 apparaît sous la plume d'un scribe peu expert, qui n'est pas notaire de formation mais prêtre et protopape, et dont le grec est d'un niveau rien moins que littéraire : on peut estimer que cette mention correspond à un usage réel du terme dans le langage quotidien, et probablement d'origine byzantine, sans solution de continuité avec l'époque dite «normande ».

35 Peters-Custot 2009 a, p. 106-107. L'expression apparaît notamment dans la Vie des sains Christophore, Sabas et Macaire : Cozza-Luzi (éd.) 1893, p. 35 et 82. L'expression peut avoir un sens administratif et non monastique : signalons en effet qu'un testament grec d'époque byzantine (1041) et présent dans les archives de Carbone (Robinson 1928-1930, II), mentionne une « éparchie du Latinianon ». Dans la mesure où le Latinianon était, comme on l'a vu, un ancien gastaldat longobard, il est possible que ce nom ait été repris de celui du chef-lieu, ce qui ferait de l'éparchie du Latinianon la diakratèsis dudit bourg.

36 On se rapporte au fameux passage de la relation de la seconde ambassade de Liutprand de Crémone à Constantinople, dans laquelle l'évêque expose que l'empereur Nicéphore Phocas commanda au patriarche diverses modifications de la carte épiscopale de l'Italie byzantine, et notamment, pour ce qui nous concerne, la fondation de l'évêché de Tursi, en Basilicate méridionale : Scripsit itaque [sur ordre de Nicéphore II] Polyeuctos Constantinopolitanus patriarcha privilegium Hydrontino episcopo, quatinus sua auctoritate habeat licentiam episcopos consecrandi in Acirentila, Turcico, Gravina, Maceria, Tricario, qui ad consecrationem domini apostolici pertinere videntur, dans Chiesa (éd.) 1998, §62 p. 215, et désormais Bougard 2015, p. 416. Un stratège de Lucanie, qui fait une donation de 1000 pieds de vigne, est attesté par un seul document de 1042 : Guillou (éd.) 1967, n 4 . La création de ce thème (qui n'a pas duré) doit être mise en rapport avec l'action du prince de Salerne Guaimar V, qui se proclame à ce moment dux Apuliae et Calabriae; voir Martin 2006, p. 312.

37 La concession de l'archimandritat à S. Elia di Carbone est le fait du roi Guillaume II : Robinson 1928-1930, n XLVI-94, et Holtzmann 1956, nº 8. Voir Kölzer (éd.) 1983, p. 15 (Kölzer [éd.] 1990, p. 13).

38 Voir la carte en appendice de cet article.

39 A ce sujet voir Falkenhausen 1983 ; Falkenhausen 1994 a ; Rognoni (éd.) 2004, p. 13-19.

40 Peters-Custot 2002. La transcription du document est due à Jean-Marie Martin.

41 Ibid., p. 1052-1060.

42 Enzensberger 1973 ; Peters-Custot 2009 a, p. 463-472.

43 Petta 1971, p. $63, n^{\circ} 31$ et p. $64 n^{\circ} 48$.

44 Laurent-Guillou 1960, p. 152 note 4.

45 Ibid., p. 152-158.

46 Ibid., p. 154-155. L'inventaire fait notamment état de nombreux ouvrages de patristique (Jean Chrysostome, Basile, Grégoire de Naziance, Jean Climaque, etc.).

47 Voir en particulier Falkenhausen 1994 b.

48 Santoro 1601. Ughelli-Coleti 1717-1722, VII, c. 71-80. Cotroneo 1902. Robinson 1928-1930. Holtzmann 1956. Breccia 1991.

49 Voir Martin 1985-1986 ; Peters-Custot 2006 ; Falkenhausen 1994 b, p. 62, n. 6-7. 
50 Archivio Doria Pamphili, 23 (éd. Robinson 1928-1930 n XIV, 1095/96) ; $n^{\circ} 6$ (éd. Holtzmann $1956 n^{\circ} 4$ (1104); $n^{\circ} 38$ (Holtzmann $\left.1956 n^{\circ} 5,1108\right)$; n 66 a (éd. Robinson 1928/1930 $\mathrm{n}^{\circ}$ XVIII, 1112).

51 Breccia, Fugaro 1994, p. 10 et n. 8.

52 Dans l'ordre : Archivio Doria Pamphili, $n^{\circ} 5$ a (Robinson 1928-1930 n ${ }^{\circ}$ XLV) ; 11 (Holtzmann $1956 \mathrm{n}^{\circ}$ 9) ; 42 (Robinson 1928)1930 n L) ; voir Breccia, Fugaro 1994, p. 10 et n. 21-25.

53 Breccia, Fugaro 1994, p. 143-147.

54 Falkenhausen 1994 b, p. 67-69.

55 Holtzmann 1956, n 1 ; voir Falkenhausen 1994 b, p. 70-73.

56 Archivio Doria Pamphili, $\mathrm{n}^{\text {os }} 53$, 59, 61, 72, 76, 77, 84, 88, 89, 93, 95, 97, 99, 102, éd. Robinson 1928-1930, n ${ }^{\text {ss }}$ IV, X, XII, XXV, XXVII, XXVIII, XXXVI, IX, XL a, XLIV, XLIX, LII, LIV, LXIV.

57 Voir Falkenhausen 1994 b, p. 82.

58 Ibid. , p. 82 et n. 171. Gastone Breccia insiste, de même, sur le fait que divers aspects de l'écriture des parchemins de Carbone sont semblables à l'écriture des notaires tarentins : Breccia 1997, p. 88. Voir Peters-Custot 2009 a.

59 Voir Robinson 1928-1930, n $^{\text {s }}$ XLVIII, L, LVI a (1170-1183).

60 Breccia, Fugaro 1994, p. 33-34.

61 Petta 1972, p. 153.

62 Robinson 1928-1930, I, p. 307.

63 Voir Laurent-Guillou 1960 p. 154-156. Batiffol 1891, p. 120-122, Petta 1972, p. 156 : dans la seconde moitié du XVII ${ }^{\mathrm{e}}$ siècle, la bibliothèque (transférée à S. Basilio de Rome) possédait encore 88 livres (102 au XV d'après Athanase Chalkéopoulos). Mercati 1935. Batiffol 1889 a. Vaccari 1925, p. 308-309: scribes de Carbone. On trouvera une liste des manuscrits du Vatican et de Grottaferrata provenant de Carbone dans Petta 1994, p. 98. La Bibliothèque Vaticane conserve notamment un euchologe de Carbone (cod. Vat. Gr. 2005), fait après la mort du roi Tancrède : voir Falkenhausen 1994 b, p. 80 et 87 ; Jacob 1995.

64 Voir Poncet 2004.

65 Holtzmann 1956, p. 39.

66 Robinson1928-1930, I, p. 318.

67 Holtzmann 1956, p. 37-38.

68 Selon G. Robinson (Robinson 1928-1930, I, p. 313), dans les années 1540, l'abbé commendataire Ferdinando Ruggieri (1540-1542) avait présenté à Naples des actes de Bohémond II, Richard le Sénéchal, Alexandre et Richard de Chiaromonte, trouvés à la chartreuse de Padula et traduits en latin, à sa demande, par les Napolitains Giampaolo Vernalione et Vittorio Tarentino.

69 Santoro 1601. La Bibliothèque Vaticane possède deux copies originales du volume, qui a en outre été traduit en italien en 1831 : Holtzmann 1956, p. 34 note 1.

70 Augusta Acconcia Longo (Acconcia Longo 1994) dénonce l'assimilation de Luc Carbuni, fondateur de Carbone (et disciple de saint Sabas) avec Luc d'Armento (également disciple de Sabas), s'appuyant notamment sur le document $\mathrm{n}^{\circ}$ VII (de 1059) édité par Robinson 1928-1930.

71 Holtzmann 1956, n 1 , p. 40-42.

72 Ughelli-Coleti 1717-1722.

73 Pirro 1733.

74 Voir Breccia 1991.

75 Archivio Segreto Vaticano, fondo Basiliani, t. I

76 Holtzmann 1956, p. 38.

77 Basiliani $1, \mathrm{f}^{\circ} 86 \mathrm{v}$.

78 Batiffol 1889 b. Minisci 1952.

79 Batiffol 1888. 
80 Summa bullarum et constitutionum apostolicarum pro ordine S. P. Basilii Magni, aliorumque Collectaneorum eumdem ordinem spectantium a P. D. Petro Menniti eiusdem Ordinis Abbatis Generalis digesta, et conscripta, anno MDCCVII ; la chronique porte le titre : Cronica del Monastero Archimandritale di S. Elia di Carbone dell'ordine di S. Basilio Magno. Nous n'avons pas encore examiné le volume 23.

81 Breccia 1991, p. 33-34 et en particulier p. 33 note 60.

82 Ibid., $\mathrm{n}^{\text {os }} 20$ et 21, respectivement de 1572 et 1594 (Basiliani I, fo $126 \mathrm{r}$ et 127r).

83 Ils figurent dans le Catalogo delle pergamene esistenti nel nostro archivio, compilé en 1883 par Rocchi : Breccia 1991, p. 41.

84 Cod. Vat. Lat. 13118 , perg. 3.

85 Breccia 1991, n 3, p. 71-75 (1105), dont une autre version a été publiée par Holtzmann ; $\mathrm{n}^{\circ} 4$, p. $75-77$ (1134); n 8, p. 83 (1361); n 11, p. 90-94 (1399).

86 Santoro 1831.

87 Huillard-Bréholles 1859-1861, IV-1, p. 387-389.

88 Winkelmann 1880-1885 I, $\mathrm{n}^{\circ} 172$.

89 Cotroneo 1898 p. 390-391. Cotroneo 1902 p. 40-42.

90 Minieri Riccio 1878, $\mathrm{n}^{\circ}$ XXXVII, p. 283-284.

91 Gastone Breccia (Breccia 1994, p. 141-142) publie un mandement de Charles II (Aversa, 29 juillet 1305) dont une copie est conservée à l'Archivio di Stato de Naples (Cappellano Maggiore. Processi di Regio Patronato, vol. 1046, nº 94, p. 52v-53).

92 Robinson 1928-1930. Le mot «cartulary » est employé abusivement pour désigner le chartrier conservé à l'Archivio Doria Pamphili.

93 Holtzmann 1956 (p. 39) n'en compte que 68, mais il y a des numéros doubles.

94 On trouve dans Fonseca-Lerra (éd.) 1994 la photographie de la première page d'une « regia platea $\gg$ de 1741.

95 Holtzmann 1956, p. 36.

96 Archivio paleografico italiano 1954, tav. 29-30.

97 Cappelli 1941-1942, seconde partie, p. 33-42.

98 Enzensberger (éd.) 1996, n 2 . Zielinski (éd.) 1982, n 19. Kölzer (éd.) 1983 et Kölzer (éd.) $1990, n^{\text {os }} 4,33,35$; à propos du n ${ }^{\circ} 4$ (p. 15 de 1983, p. 13 de 1990), cet auteur fait une mise au point sur la création de l'archimandritat. Koch (éd.) 2010, n 587 (rééd. de l'acte publié par Winkelmann).

99 Peters-Custot 2002, p. 1065-1066 (Archivio Doria Pamphili, 43, du 14 janvier 1315).

100 Garufi 1902, n 270 p. 153 (1310), n 21 p. 205 . Vendola 1939, n 2069 p. 161 . Hoberg 1949, p. $200(1402,1431,1432)$.

101 Breccia 1994, p. 143-147. On trouve encore, dans Breccia 1997, une série de photographies (tav. $1 \mathrm{a}-8$ b) des documents 54, 61, 62, 63, 64, 72, 76, 79, 81, 83, 95, 99, 101, 109 conservés à l'Archivio Doria Pamphili.

102 Breccia, Fugaro 1994.

\section{Pour citer cet article}

Référence électronique

Adele Di Lorenzo, Jean-Marie Martin et Annick Peters-Custot, « Le monastère de S. Elia di Carbone, ses archives et l'histoire de la Basilicate médiévale », Mélanges de l'École française de Rome - Moyen $\hat{A}$ ge [En ligne], 128-2। 2016, mis en ligne le 07 septembre 2016, consulté le 07 septembre 2016. URL: http://mefrm.revues.org/3318

\section{À propos des auteurs}

Adele Di Lorenzo

CNRS - UMR Orient et Méditerranée - adelenrica@gmail.com

Jean-Marie Martin

CNRS - UMR Orient et Méditerranée - martin-hisard@wanadoo.fr 
Annick Peters-Custot

Université de Nantes, C.R.H.I.A. (E.A. 1163) - annick@ peters-custot.fr

\section{Droits d'auteur}

(c) École française de Rome

\section{Résumés}

Le monastère grec de St-Élie et St-Anastase de Carbone a été fondé à la fin du $\mathrm{X}^{\mathrm{e}}$ siècle lorsque des moines grecs venus de Calabre se sont installés en Basilicate. Le roi Guillaume II fait en 1168 de son abbé l'archimandrite des monastères grecs de Basilicate. L'abbaye décline aux XIII ${ }^{\mathrm{e}}$ et $\mathrm{XIV}^{\mathrm{e}}$ siècles, perd peu à peu son caractère grec, passe en 1474 sous le régime de la commende. Les abbés commendataires Giulio Antonio Santoro, Paolo Emilio Santoro et Giovanni Battista Pamphili, qui se succèdent de 1570 à 1644, puis l'abbé général des Basiliens Pietro Menniti à la fin du XVII ${ }^{\mathrm{e}}$ siècle ont permis de conserver de nombreux documents, grecs et latins, du $\mathrm{XI}^{\mathrm{e}}$ siècle au début de l'époque moderne ; ces documents (originaux, copies, traductions) sont aujourd'hui dispersés entre l'Archivio Doria Pamphili (Rome), le fonds Basiliani de l'Archivio Segreto Vaticano, quelques autres fonds, ou conservés dans des éditions anciennes, notamment dans le livre de P. E. Santoro. La partie ancienne du fonds a été publiée en 1928-1930 par Gertrude Robinson, de façon peu satisfaisante ; Walther Holtzmann, puis Gastone Breccia en ont aussi édité des éléments. On se propose de donner une édition scientifique des actes grecs et latins de ce fonds bilingue, le plus important pour l'histoire de la Basilicate médiévale et du monachisme grec en Italie.

The Greek monastery of St-Elias and St-Anastasius of Carbone was founded at the end of the 10th century, when Greek monks came from Calabria and set up in Basilicata. King William II promoted its abbot to archimandrite of the Greek monasteries of Basilicata. It declined in the 13th and 14th centuries, gradually lost its Greek character and since 1474 was submitted to commendatory abbots. The commendatory abbots Giulio Antonio Santoro, Paolo Emilio Santoro and Giovanni Battista Pamphili, who ruled the monastery from 1570 to 1644, and Pietro Menniti, general abbot of the Basilian order at the end of the 17th century, permitted to preserve many Greek and Latin deeds from the 11th century to the beginning of the modern age ; these documents (originals, copies, translations) are scattered in the Archivio Doria Pamphili (Rome), the fondo Basiliani of the Archivio Segreto Vaticano and other archives, or preserved in ancient editions (in particular in the book of P. E. Santoro). The oldest part of the collection was published in 1928-1930 by Gertrude Robinson, but in a very unsatisfactory edition. Walther Holtzmann and Gastone Breccia published some deeds. We propose to give a complete scientific edition of all the Greek and Latin deeds of this bilingual collection, the most important for the history of medieval Basilicata and of the Greek monasticism in Italy.

\section{Entrées d'index}

Mots-clés : Archimandritat, Basilicate, monachisme grec, Ordre basilien Keywords : Archimandrite, Basilian Order, Basilicata, Greek monachism 